\title{
The bindosome is a structural component of the Sulfolobus solfataricus cell envelope
}

\author{
Benham Zolghadr • Andreas Klingl • \\ Reinard Rachel • Arnold J. M. Driessen • \\ Sonja-Verena Albers
}

Received: 4 May 2010/Accepted: 22 December 2010/Published online: 14 January 2011

(C) The Author(s) 2011. This article is published with open access at Springerlink.com

\begin{abstract}
Sugar binding proteins of the thermoacidophile Sulfolobus solfataricus function together with $\mathrm{ABC}$ transporters in the uptake of sugars. They are synthesized as precursors with a class III signal peptide that are normally found in archaeal flagellins and bacterial type IV pilins. The functional expression of sugar binding proteins at the cell surface is dependent on the bindosome assembly system (Bas) that is homologous to bacterial type IV pilin assembly systems. The Bas system consists of an assembly ATPase, BasE; a membrane anchoring protein, BasF; and three small class III signal peptide containing proteins BasABC. Expression of BasEF in a $S$. solfataricus $\triangle b a s E F$ strain restored the uptake of glucose, while an ATPase mutant of BasE was unable to complement. BasEF was detergent-extracted from $S$. solfataricus membranes as a stable protein complex. Solute binding proteins can be extracted from the cell surface as two high molecular mass complexes of 600 and $400 \mathrm{kDa}$, wherein the largest complex also contains the main S-layer protein SlaA. Electron
\end{abstract}

Communicated by L. Huang.

B. Zolghadr · S.-V. Albers $(\square)$

Molecular Biology of Archaea, Max-Planck-Institute

for Terrestrial Microbiology, Karl-von-Frisch-Strasse 10, 35043 Marburg, Germany

e-mail: albers@mpi-marburg.mpg.de

\section{A. Klingl · R. Rachel}

Centre for Electron Microscopy, Institute for Microbiology,

Universität Regensburg, Universitätsstr. 31,

93053 Regensburg, Germany

\section{B. Zolghadr · A. J. M. Driessen}

Department of Microbiology, Groningen Biomolecular Sciences and Biotechnology Institute, The Zernike Institute for Advanced Materials, University of Groningen, Kerklaan 30,

9751 NN Haren, The Netherlands microscopic analysis of the cell surface of the wild-type and $\triangle b a s E F$ strain indicates that the absence of the BasEF complex causes an alteration in cell morphology and the corrugation of the S-layer pattern that is reversed by complementation with the BasEF complex. These results suggest an interaction between the S-layer and the sugar binding proteins that contribute to cell shape.

Keywords Cell envelope $\cdot$ Archaea $\cdot$ Sulfolobus $\cdot$ S-layer

\section{Introduction}

Sulfolobus solfataricus is a thermophilic crenarchaeon belonging to the family of Sulfolobales and it optimally grows at $80^{\circ} \mathrm{C}$ and at a $\mathrm{pH}$ value of 3-4. It was originally isolated from sulfur-rich volcanic areas in the USA, Italy and Iceland and its metabolism and physiology is adapted to these environments (Zillig et al. 1980). From the different strains from Sulfolobales that have been tested for their ability to grow on various minimal media of sugars such as glucose, arabinose and disaccharides such as cellobiose, maltose and lactose, only S. solfataricus was found to grow on minimal sugar media (Grogan 1989). The natural environment of $S$. solfataricus is relatively poor in substrates, which necessitates efficient mechanisms of substrate transport across the membrane. In recent years, the ABC transporters in $S$. solfataricus have been studied extensively and several sugar binding proteins were identified that bind sugars at subnanomolar concentrations (Albers et al. 1999a; Elferink et al. 2001).

GlcS is a binding protein that binds glucose and galactose, while AraS binds arabinose, fructose and xylose. In bacteria, binding proteins are directed to the extracellular compartment by means of an $\mathrm{N}$-terminal signal peptide that 
upon translocation across the cytoplasmic membrane is removed by a signal peptidase. However, sequence analysis of GlcS and AraS showed that these proteins bear a type IV pilin-like signal peptide or class III signal peptide at their N-terminus (Albers et al. 1999b; Albers and Driessen 2002). Class III signal sequence bearing proteins are normally assembled into an extracellular macromolecular complex such as the pilus or flagellar structures. Class III signal peptides are cleaved by a dedicated type IV signal peptidase which upon processing removes only the positively charged N-terminus leaving a hydrophobic domain of about 20 amino acids attached at the N-terminus of the mature protein (Albers and Driessen 2002; Craig et al. 2004). By the removal of the positive charges, translocation of the protein across the cytoplasmic membrane is possible whereupon the hydrophobic N-terminus acts as scaffold for the assembly of the protein into a supramolecular structure at the extracellular surface of the membrane. In vitro cleavage assays showed that the class III signal peptides of GlcS and AraS are indeed processed by the type IV signal peptidase of S. solfataricus $\mathrm{PibD}$ (Albers et al. 2003). Importantly, the presence of the class III signal peptides predicts that an assembly system is required for the functional expression of the sugar binding proteins at the cell surface. S. solfataricus contains five operons that encode subunits that are homologous to components of bacterial type IV pili assembly systems. Recently, we have shown that the Bas system of $S$. solfataricus is responsible for the functional expression of sugar binding proteins in the cell envelope (Zolghadr et al. 2007). The Bas system consists of the assembly ATPase, BasE; a membrane protein, BasF; and three small proteins BasABC that are synthesized with a class III signal peptide and that possibly form a pseudopilus-like structure. Deletion of the basEF and basABC genes results in a growth deficiency on various sugars that for uptake depend on a class III signal peptide bearing sugar binding protein. Although the uptake of glucose was impaired in the $\triangle b a s E F$ and $\triangle$ bas $A B C$ mutants, the translocation of the binding proteins across the cytoplasmic membrane seemed unaffected suggesting that the Bas system may be involved in correct localization of the binding proteins.

BasE belongs to the family of type IV pili assembly/type II secretion ATPases (Planet et al. 2001; Peabody et al. 2003; Albers and Driessen 2005) that share several conserved motifs in the Walker A and B sites, and the Asp and His Box boxes. In the bacterial ATPases, these conserved motifs are located at the C-terminal domain (CTD) that constitutes the ATP binding and hydrolysis site (Possot and Pugsley 1994; Sandkvist 2001). BasF is a membrane protein that due to its similarity with the EspN protein of the type II secretion system of Vibrio cholerae (Abendroth et al. 2005) is likely to interact with BasE to constitute the functional assembly system.
Currently, the precise role of the Bas system in the localization of the sugar binding proteins remains unclear. We now show that the uptake of glucose is directly dependent on the ATPase activity of BasE, and that BasE and $\mathrm{BasF}$ are interacting proteins. Localization studies indicate that the sugar binding proteins are contained in high molecular mass complexes that are associated with the S-layer. The deletion of the basEF genes does not interfere with complex formation but results in cells with an altered morphology and a distorted S-layer. These data indicate that sugar binding proteins are functionally associated with the S-layer.

\section{Experimental methods}

\section{S. solfataricus growth conditions}

S. solfataricus PBL2025 (Schelert et al. 2004) and the $\triangle$ basEF strain (Zolghadr et al. 2007) were grown in Brock medium at $\mathrm{pH} 3$ and $80^{\circ} \mathrm{C}$ (Brock et al. 1972) and the medium was supplemented with $0.1 \%$ (w/v) of tryptone as sole carbon and energy source. Growth of cells was monitored by measuring the optical density at $600 \mathrm{~nm}$.

\section{Construction of the basE mutant}

The lysine residue 237 in the Walker A site of BasE was replaced by an alanine by site-directed mutagenesis. The pMZ11 plasmid harboring the basEF genes (pSVA69) was used as a template for the generation of the mutation using the primers $5^{\prime}$-ACGACATTTAATATAAGAGCATTCCC TGAA- $3^{\prime}$ and $5^{\prime}$-TTTTCAGGGAATGCTCTTATATTAA ATGTC-3'. The mutated basEF was transferred to the SSV1 virus vector with $B \ln \mathrm{I}$ and EagI restriction sites as described, yielding the plasmid pSVA107 (Zolghadr et al. 2007).

\section{Glucose uptake assays}

The uptake of $\mathrm{D}-\left[{ }^{14} \mathrm{C}\right]$-glucose $(250 \mathrm{Ci} / \mathrm{mmol})$ by $S$. solfataricus cells was measured as described (Albers et al. 1999a).

Isolation of cell envelope

Cells were harvested at $\mathrm{OD}_{600}$ of $\sim 1$ and resuspended in a buffer containing $50 \mathrm{mM}$ Hepes/ $\mathrm{NaOH}$ at $\mathrm{pH} 8$ and supplemented with $1 \mathrm{mM}$ PMSF and a small amount of DNase I. Cells were broken by 10 cycles of $10 \mathrm{~s}$ sonication and $50 \mathrm{~s}$ rest (Soniprep 150, LA Abcoude). Unbroken cells were removed by low spin centrifugation at 4,300 rpm and $4^{\circ} \mathrm{C}$ for $15 \mathrm{~min}$, and cell envelopes were harvested by 
ultracentrifugation at $100,000 \times g$ for $1 \mathrm{~h}$ at $4^{\circ} \mathrm{C}$. Cell envelope pellets were resuspended in $50 \mathrm{mM}$ Hepes/ $\mathrm{NaOH}$ buffer at $\mathrm{pH} 8$ and frozen in liquid nitrogen and stored at $-80^{\circ} \mathrm{C}$. Cell envelopes included the cytoplasmic membrane as well as the S-layer and all other extracellular cell attached components.

\section{Purification of BasE/F from Sulfolobus cell envelopes}

BasE and BasF were co-expressed in the $S$. solfataricus $\triangle$ basEF strain using pSVA75 (Zolghadr et al. 2007) with a C-terminal tandem tag (Strep-tag and $10 \times$ His-tag) on BasF. Cell envelopes from the expression strain were isolated as described above and solubilized in buffer $\mathrm{A}$ (50 mM Hepes buffer at $\mathrm{pH}$ 8) supplemented with $2 \%$ DDM for $1 \mathrm{~h}$ at room temperature. Solubilized membrane proteins were separated from non-solubilized membranes by ultracentrifugation $\left(100,000 \times g\right.$ for $1 \mathrm{~h}$ at $\left.4^{\circ} \mathrm{C}\right)$, and the supernatant fraction was applied to a nickel NTA affinity column. Unbound proteins were eluted with $50 \mathrm{mM}$ imidazole and specifically bound proteins were eluted with $200 \mathrm{mM}$ imidazole in buffer A.

For size exclusion chromatography analysis of the BasEF complex, a Superdex 200 column was equilibrated with $50 \mathrm{mM}$ Hepes/ $\mathrm{NaOH}$, pH 8 supplemented with $0.05 \%$ (w/v) DDM. The chromatography was run with same buffer at $1 \mathrm{ml} / \mathrm{min}$ on a AKTA purifier (GHealthcare) and the fractions were collected at $1 \mathrm{ml}$ per fraction. BasE and BasF were detected by Western blotting using anti-BasE and anti-BasF antibodies. BasF antibodies were generated against a synthetic peptide (QKYKSRAREIRDDIC) of BasF in rabbits and BasE antibodies against purified BasE (Genescript).

Purification of binding proteins

by ConA chromatography

Isolated cell envelopes from $S$. solfataricus PBL2025 and $\triangle$ basEF cells were solubilized in buffer A (20 mM Hepes buffer at $\mathrm{pH} 7.5,100 \mathrm{mM} \mathrm{NaCl}$ ) supplemented with $2 \%$ DDM for $1 \mathrm{~h}$ at room temperature. The solubilized fraction was separated from non-solubilized membranes by ultracentrifugation at $100,000 \times g$ for $1 \mathrm{~h}$ at $4^{\circ} \mathrm{C}$. Solubilized membrane proteins were passed onto a $1 \mathrm{ml}$ ConA Sepharose column equilibrated with $10 \mathrm{ml}$ buffer $\mathrm{A}$. The column was washed with $30 \mathrm{ml}$ buffer $\mathrm{A}$, and bound membrane proteins were eluted with buffer A supplemented with $0.05 \%$ DDM and $150 \mathrm{mM}$ methyl- $\alpha$-D-mannopyranoside. The flow through, wash steps and elution fractions were analyzed on $12 \%$ SDS-PAGE gel. The uptake of $\mathrm{D}$ - $\left[{ }^{14} \mathrm{C}\right]$-glucose $(250 \mathrm{Ci} / \mathrm{mmol})$ by $S$. solfataricus cells was measured as discussed previously (Albers et al. 1999a).
Fixation, freeze substitution and embedding of $S$. solfataricus cells

High-pressure freezing of samples was carried out with high-pressure freezer (Leica EM-PACT 2) as described previously (Rachel et al. 2002). The samples were freeze substituted in a Leica EM AFS (automatic freeze substitution system, Leica, Vienna, Austria) with pure acetone containing $2 \%(\mathrm{w} / \mathrm{v})$ osmium tetroxide and $0.1 \%(\mathrm{w} / \mathrm{v})$ uranyl acetate, and $5 \% \mathrm{H}_{2} \mathrm{O}$ at $-90^{\circ} \mathrm{C}$ for $72 \mathrm{~h},-60^{\circ} \mathrm{C}$ for $8 \mathrm{~h}$ and $-30^{\circ} \mathrm{C}$ for $6 \mathrm{~h}$. Samples were washed for $1 \mathrm{~h}$ in acetone at room temperature and infiltrated in Epon 812 (Ted Pella, Inc., USA) for $30 \mathrm{~h}$ and the Epon was polymerized at $60^{\circ} \mathrm{C}$ for $24 \mathrm{~h}$. Ultrathin sections were cut with a Leica UCT ultramicrotome and mounted on carboncoated copper grids. Transmission electron micrographs were recorded using a slow-scan CCD camera (TVIPS, Gauting, Germany) on a Philips CM 12 (FEI, Eindhoven, The Netherlands) operated at $120 \mathrm{kV}$, which is also equipped with a Gatan TV 673 wide-angle camera (Gatan, München) for searching and focusing.

Freeze-etching of S. solfataricus cells

Cells were harvested by centrifugation at $5,000 \mathrm{rpm}$ for $15 \mathrm{~min}$ and a $1.5-\mu \mathrm{l}$ aliquot of the concentrated cell suspension was loaded into a gold-carrier and frozen in liquid nitrogen. The sample was cut with a cold knife $(T \leq$ $\left.-185^{\circ} \mathrm{C}\right)$ and etched for $4 \mathrm{~min}\left(T=-97^{\circ} \mathrm{C} ; p \leq 1.3 \times\right.$ $10^{-5} \mathrm{~Pa}$ ) in a CFE-50 freeze-etch unit (Cressington, Watford, UK), The freeze-etched sample was shadowed

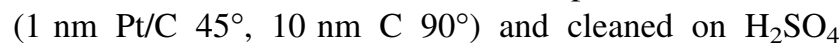
(70\%). Replicas were picked up with 700 mesh hexagonal copper grids. Transmission electron micrographs were taken as above.

\section{Results}

Characterization of BasEF by site-directed mutagenesis

To determine the role of the ATPase activity of BasE, the conserved lysine residue (K237) of the Walker A motif was replaced by an alanine residue by site-directed mutagenesis. In general, the introduction of this mutation in homologous assembly ATPases results in a loss of the ATPase activity and a functional defect (Herdendorf et al. 2002; Sexton et al. 2004). The mutated basE gene was subcloned with basF into the virus expression vector pSVA9 of $S$. solfataricus to yield pSVA107. The pSVA107 plasmid was introduced into the $\triangle$ basEF strain and glucose uptake was measured and compared to a strain transformed with pSVA75 containing the native basEF genes (Zolghadr 
et al. 2007; Albers et al. 1999a). The cells complemented with $\mathrm{BasE}(\mathrm{K} 237 \mathrm{~A}) \mathrm{F}$ showed a glucose transport activity similar to the negative control, whereas cells transformed with the wild-type BasEF showed high levels of glucose uptake (Fig. 1). The results demonstrate that the ATPase activity of BasE is essential for active glucose transport in S. solfataricus.

\section{BasE and BasF co-purified as a complex}

Since the expression of BasEF in the $\triangle b a s E F$ strain restored the glucose uptake, we performed a detergent extraction and purification of the Bas complex from these cells in order to determine the complex subunit composition. Herein, BasE and BasF were co-expressed in S. solfataricus cell envelopes with a C-terminal tandem tag (Strep-tag/10 $\times$ His-tag) on BasF. After solubilization BasF was purified using Histag affinity chromatography and eluted with the expected

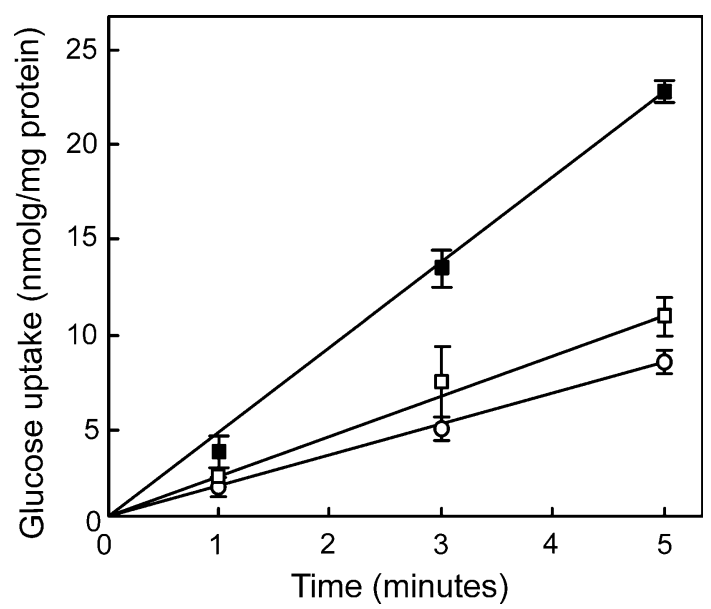

Fig. 1 Uptake of glucose by the $\triangle$ basEF strain without (open circles) and with a plasmid containing BasEF (pSVA75) (closed squares) or $\operatorname{BasE}(\mathrm{K} 237 \mathrm{~A}) \mathrm{F}$ (pSVA107) (open squares)

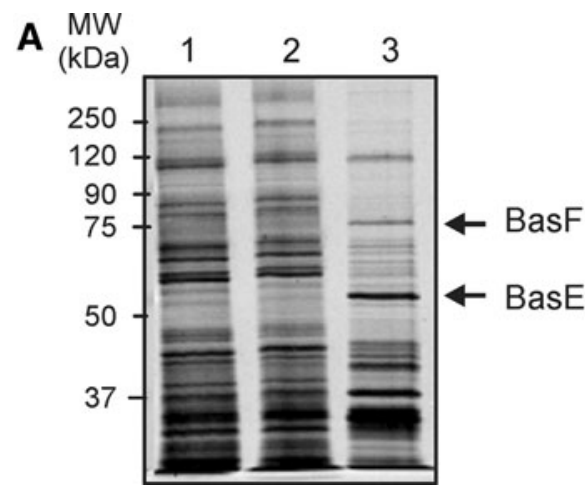

Fig. 2 Co-purification of the BasE and BasF proteins expressed in $S$. solfataricus $\triangle$ basEF cells. Isolated membranes were solubilized in 2\% DDM, and solubilized proteins were passed over a Ni-NTA affinity column and eluted with $100 \mathrm{mM}$ imidazole. Fractions were molecular size of $75 \mathrm{kDa}$ (Fig. 2a) and various other proteins. The identity of BasF was confirmed by immunoblotting using an antibody directed against BasF (Fig. 2b). The elution fraction contained another protein with a size of $\sim 60 \mathrm{kDa}$ (Fig. 2a) which was identified as BasE by immunoblotting using a polyclonal antibody raised against the purified BasE. BasF was also identified by mass spectrometry (data not shown). However, this protein band also contained tf55 $\alpha$, a subunit of the thermosome of $S$. solfataricus which has a similar mass as BasE which explains the greater band intensity of BasE as compared to BasF. These data indicate that $\mathrm{BasF}$ and BasE are interacting proteins forming a stable membrane protein complex.

To determine if BasEF is present in a high molecular mass complex, the partial purified BasEF complex was subjected to size exclusion chromatography using a Superdex 200 column and $0.1 \%$ dodecylmaltoside (Fig. 3a). Fractions were analyzed on SDS-PAGE (Fig. 3b), and by immunoblotting with BasF and BasE antibodies (Fig. 3c, d, respectively). The first elution peak with an approximate mass of $1 \mathrm{MDa}$ contained the tf55a protein which is forming octa- and nonamers hence the high molecular weight peak (Fig. 3b, lane 1; Schoehn et al. 2000). The second peak, which corresponded to a size of 100-200 kDa, contained the BasE and BasF proteins (Fig. 3b, lane 3). The third peak contained a protein with a mass of approximate $40 \mathrm{kDa}$ (Fig. 3b, lane 4). Mass spectroscopy analysis failed to reveal the identity of $40 \mathrm{kDa}$ protein. The estimated size of the BasEF elution fraction was smaller than expected. A stoichiometric complex of BasEF is predicted to have a molecular mass of $\sim 135 \mathrm{kDa}$. However, ATPases homologous to BasE form a hexameric ring (Herdendorf et al. 2002; Yamagata and Tainer 2007), and thus a putative BasE hexamer alone would correspond to a mass of $\sim 360 \mathrm{kDa}$. The amount of BasF molecules in the complex is unknown, but considering that the BasF is $75 \mathrm{kDa}$ while taking the

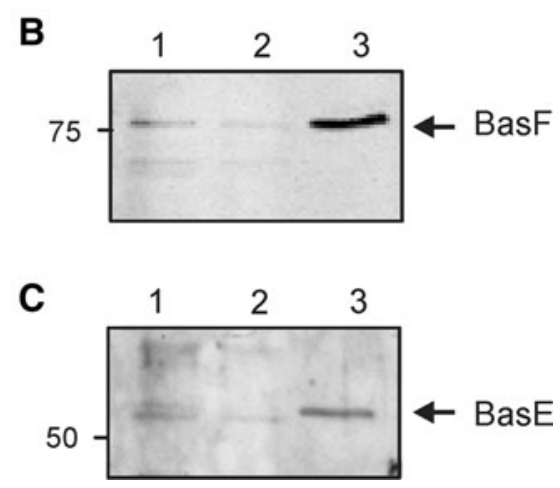

analyzed on SDS-PAGE (a), and by Western blotting using antibodies against BasF (b) and BasE (c). Start material, flow through and the $200 \mathrm{mM}$ imidazole elution fraction were loaded in lanes 1, 2 and 3, respectively 
Fig. 3 Size exclusion analysis of the elution fraction from the Ni-NTA affinity purification of the BasEF complex (a). Collected fractions were analyzed on SDS-PAGE (b) and Western blotting using antibodies against $\mathrm{BasF}(\mathbf{c})$ and BasE (d)
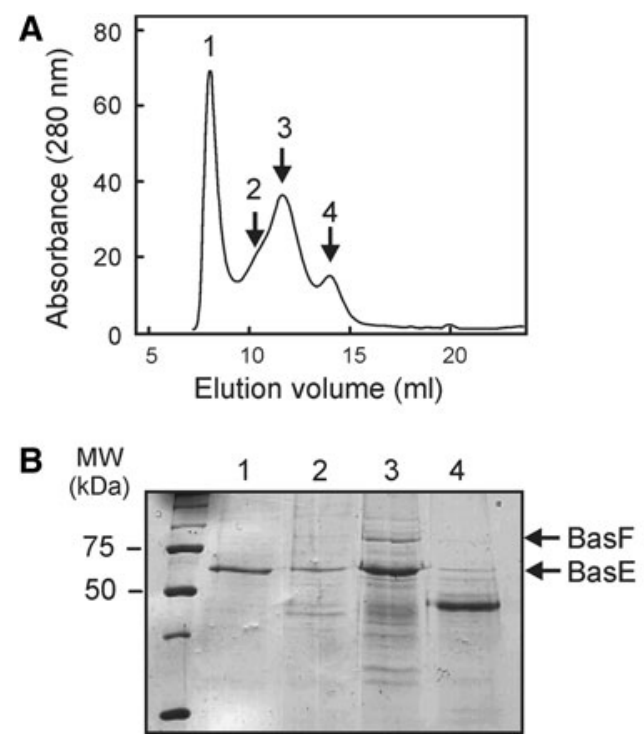
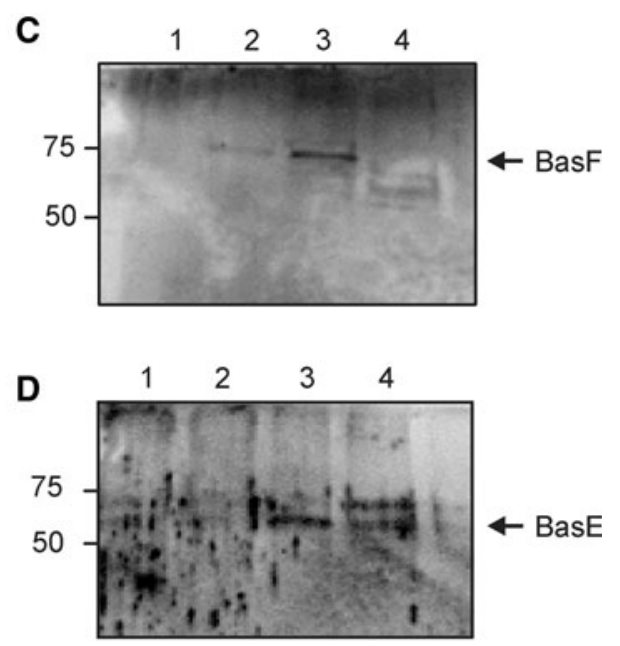

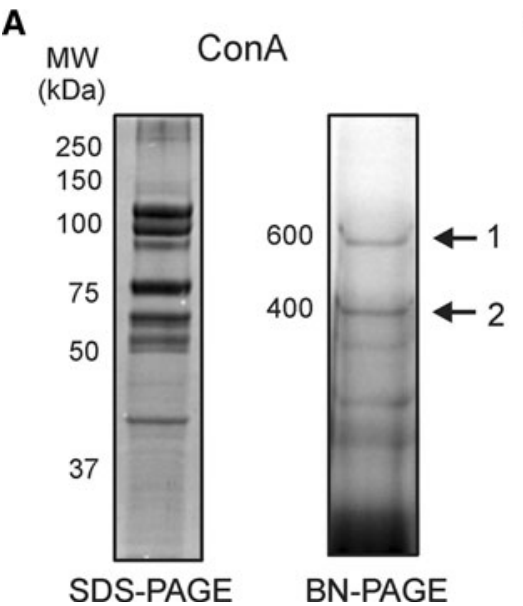

Fig. 4 Analysis of the sugar binding proteins extracted from membranes derived from $S$. solfataricus wild-type cells grown on arabinose and purified with ConA lectin affinity purification. ConA fraction analyzed by SDS-PAGE (a, left panel) and Blue-Native PAGE (a, right panel). The sugar binding proteins were present in two complexes with molecular masses of 400 and $600 \mathrm{kDa}$,

detergent micelle into account, a minimum size of $500 \mathrm{kDa}$ is expected for the BasEF complex. The intense appearance of the BasE band in Fig. 3b (lane 3) is most likely due to the presence of trimers/dimers of tf $55 \alpha$ as identified by mass spectrometry. Therefore, the eluted fraction most likely corresponds to a stoichiometric BasEF complex.

Sugar binding proteins on $S$. solfataricus are assembled into high molecular complexes

To determine if the sugar binding proteins are contained in large complexes at the cell surface, binding proteins were extracted from cell envelopes derived from S. solfataricus

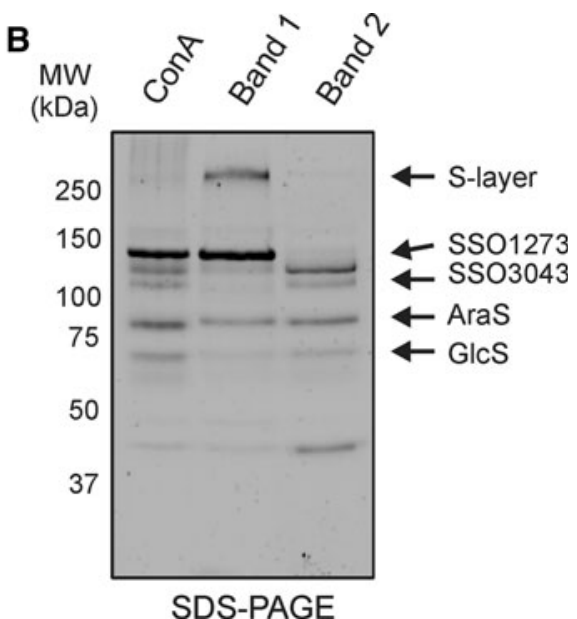

respectively. These two complexes were excised, eluted and subsequently run on an SDS-PAGE (b) to show their subunit composition. For comparison the initially isolated Con A fraction is displayed in the first lane. The identity of the proteins was determined by mass spectrometry

wild-type and $\triangle$ basEF cells using a low Triton X-100 concentration, and partially purified by ConA lectin affinity chromatography. The identity of the proteins was confirmed by mass spectrometry (Fig. 4). When analyzed by Blue-native PAGE, the ConA-enriched binding proteins were found in two main complexes with apparent molecular masses of about 600 and $400 \mathrm{kDa}$, respectively (Fig. 4). In addition, several smaller complexes were observed.

The complexes observed in the BN-PAGE showed a distinct protein composition on SDS-PAGE. Protein complex 1 contained the main S-layer protein (SlaA) and a protein at $120 \mathrm{kDa}$ that by mass spectrometry was 
Fig. 5 Analysis of the sugar binding proteins extracted from membranes derived from $S$. solfataricus wild-type and $\triangle b a s E F$ cells and purified with ConA lectin affinity purification. Isolated ConA fractions were analyzed on Blue-Native PAGE (a). Wild-type ConA fraction was analyzed by size exclusion chromatography (b), resulting in two defined peaks, which were analyzed by SDS-PAGE (c) and protein bands were identified by mass spectrometry
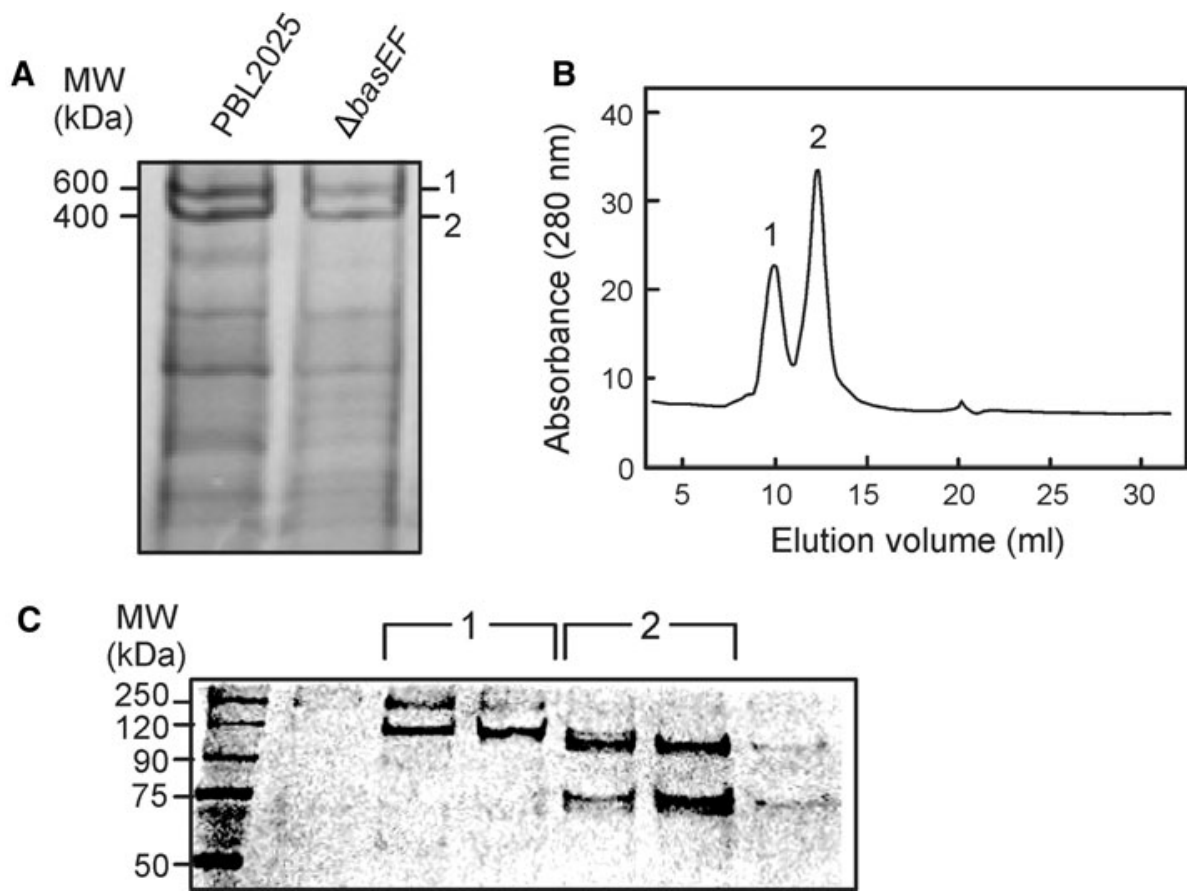

identified as a putative dipeptide binding protein, SSO1273 (Fig. 4b). SlaA has previously been detected to represent a major component of the ConA purification fraction (Ellen et al. 2009) and found to be glycosylated (data not shown). Protein complex 2 consists of the glucose binding protein (GlcS) and another putative dipeptide binding protein SSO3043 as validated by mass spectrometry (Fig. 4). Both SSO3043 and SSO1273 are synthesized with a type I signal peptide. It should be emphasized that the annotation of SSO3043 and SSO1273 as putative dipeptide binding proteins may not necessarily mean that these proteins bind dipeptides as previous analysis of such proteins revealed that most represent sugar binding proteins (Elferink et al. 2001; Nanavati et al. 2006). Especially, SSO3043 is surrounded by enzymes annotated to be involved in sugar degradation. Interestingly, when the growth medium was supplemented with $0.4 \%$ arabinose, the arabinose binding protein (AraS) was induced and found to be part of protein complex 2 on BN-PAGE (Fig. 4a, b, Band 2). These results demonstrated that the sugar binding proteins isolated from the cell surface of $S$. solfataricus are contained in high molecular mass complexes, although complex formation seems to occur independent of the Bas system.

The ConA elution fractions were also analyzed by size exclusion chromatography (Fig. 5) to estimate the native mass of these complexes. The proteins eluted in two major peaks with apparent molecular masses of 600 and $400 \mathrm{kDa}$, respectively. The protein complexes were collected and analyzed on SDS-PAGE and stained with syber ruby, a sensitive fluorescent staining method (Fig. 5b). The analysis confirms the observations made by BN-PAGE showing that the $600 \mathrm{kDa}$ complex corresponds to complex 1 with SlaA and SSO1273 as main subunits. The $400 \mathrm{kDa}$ protein complex contained GlcS and SSO3043 (Fig. 5b) and corresponds to complex 2 . These data demonstrate that the (sugar) binding proteins are contained in high molecular mass complexes with a relatively uniform size. The presence of SlaA in one of these complexes suggests that the binding proteins are associated with the S-layer.

Analysis of S. solfataricus surface layer

In an attempt to visualize the bindosome structure in the cell envelope of $S$. solfataricus, we examined the cell surface by transmission electron microscopy (TEM). Herein, cells were subjected to high-pressure freezing, freeze substitution and TEM to visualize highly defined structures of the cell envelope of the PBL2025 control strain and the $\triangle$ basEF mutant strain. TEM images showed a well-preserved cell surface from both strains in ultrathin sections (Fig. 6). Interestingly, the morphology of wildtype cells appeared as highly lobed and irregular shaped cocci, while the $\triangle b a s E F$ cells were less lobed, almost regular cocci (Fig. 6a, b). Possibly, these structural changes in the cell envelope resulted from the basEF deletion. The membrane and S-layer were clearly distinguished and enclose a quasi-periplasmic space with $25 \mathrm{~nm}$ in width (Fig. 6c, d). The periplasmic space appeared well contrasted, due to the exposure of the cells to $\mathrm{OsO}_{4}$ and uranyl acetate during the freeze substitution. This indicates the presence of an abundance of biologically relevant components. Immuno-gold labeling was performed to study the 
Fig. 6 Transmission electron microscopy of ultrathin sections of PBL2025 (a, c) and $\triangle$ basEF $(\mathbf{b}, \mathbf{d})$ cells prepared by highpressure freezing, freeze substitution and embedding in Epon. Sizes of the bars in a and b are $500 \mathrm{~nm}$, c is $200 \mathrm{~nm}$ and d is $100 \mathrm{~nm}$

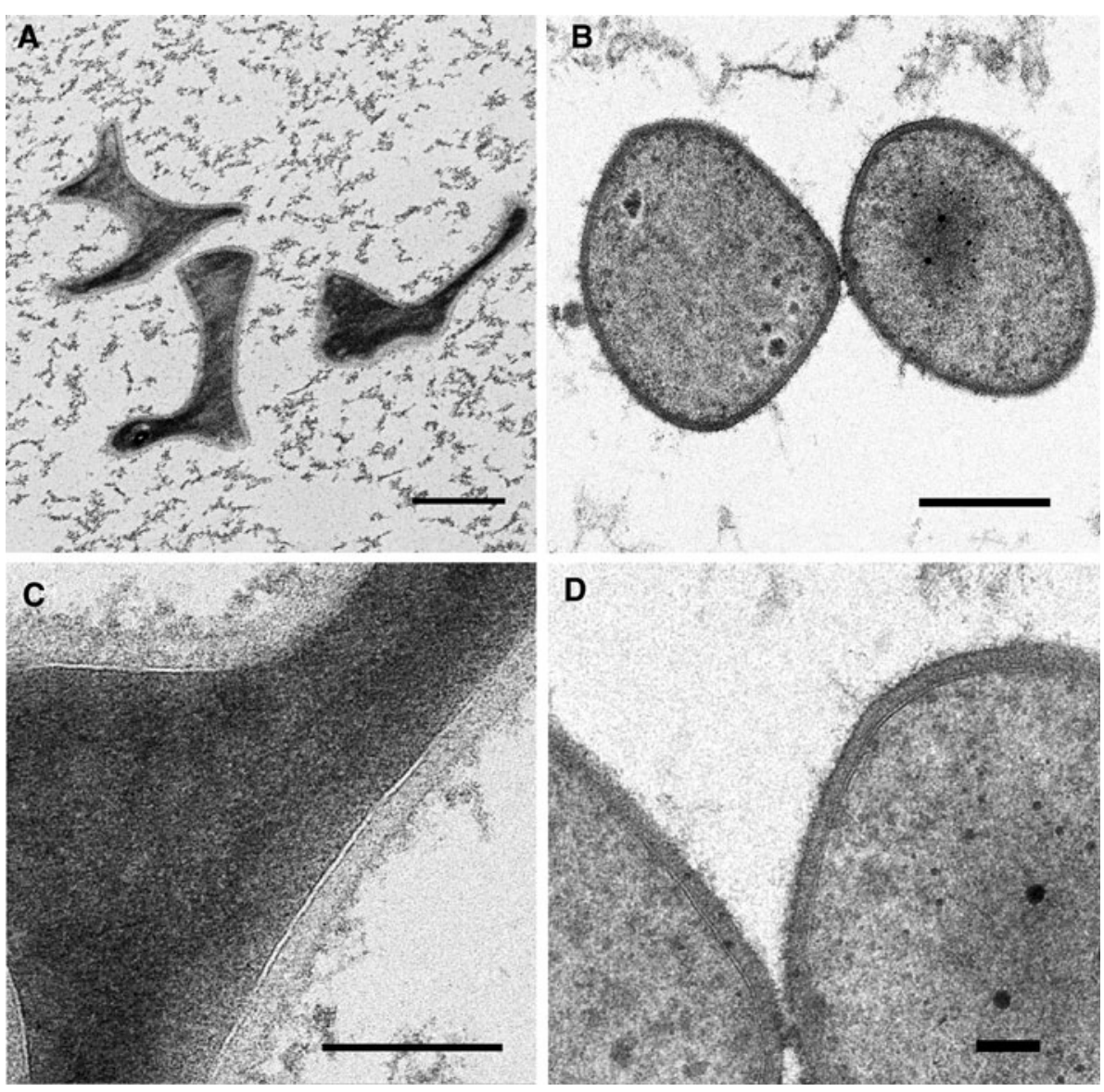

localization of binding protein in the cell envelope. However, the antibodies used to localize GlcS, the glucose binding protein, gave very poor labeling, while another antibody against the $\mathrm{A}_{1} \mathrm{~A}_{\mathrm{O}}$ ATPase (Küper et al. 2010) resulted in significant and dense labeling of the cytoplasmic membrane (data not shown). Therefore, no final conclusion about the localization of GlcS could be obtained.

In another approach, the surface layer of the PBL2025 and $\triangle b a s E F$ cells was visualized by freeze-etching freshly grown cells (Fig. 7). In these freeze-etch preparations, the S-layer of strain PBL2025 showed a crystalline lattice of pseudo-hexagonal symmetry, reflecting S-layer protein complexes with internal threefold symmetry, arranged on hexagonal lattice, in a similar manner as described (Fig. 7a, c). The crystalline lattice of the $\triangle$ basEF S-layer appeared to be less corrugated (Fig. 7b, d).

As additional evidence that the Bas system is indeed involved in determining the cell shape of $S$. solfataricus, we complemented the $\triangle b a s E F$ strain with the plasmid expressing the BasEF complex. From these cells, TEM images were obtained as described before for the wild-type and $\triangle b a s E F$ cells as well as freeze-etch preparations to visualize the S-layer. Expression of BasEF restored the lobed cell shape (Fig. 8b/d) as compared to the round $\triangle$ basEF (Fig. 6c/d). Also, the S-layer appeared like in the wild type, although disturbances were visible in the regular arrangement. These data show that the deletion of basEF results in a disturbance of the surface layer lattice, which likely causes a different cell morphology and an altered localization of the sugar binding protein complexes in the cell envelope.

\section{Discussion}

Here, we have explored several approaches to study the function of the Bas system and its role in the correct localization of the sugar binding proteins in the cell envelope of $S$. solfataricus. The Bas system plays an important role in the functional assembly of sugar transport in $S$. solfataricus. This is evident from the sugar transport defect of the basEF deletion mutant that can be complemented by the expression of BasEF. However, expression of BasE(K237A)F with a mutation of the catalytic lysine residue of the ATP binding site of BasE did not result in complementation. This implies that the ATPase activity of BasE is required for the correct localization and/or assembly of the sugar binding proteins. In a previous study, we could demonstrate that BasEF are not 
Fig. 7 Electron micrographs of freeze-etched S-layers of the PBL2025 (a, c) and $\triangle$ basEF $(\mathbf{b}, \mathbf{d})$ strains. Sizes of the bars are in $\mathbf{a}$ and $\mathbf{b}$ are $500 \mathrm{~nm}$ and in c and d $200 \mathrm{~nm}$
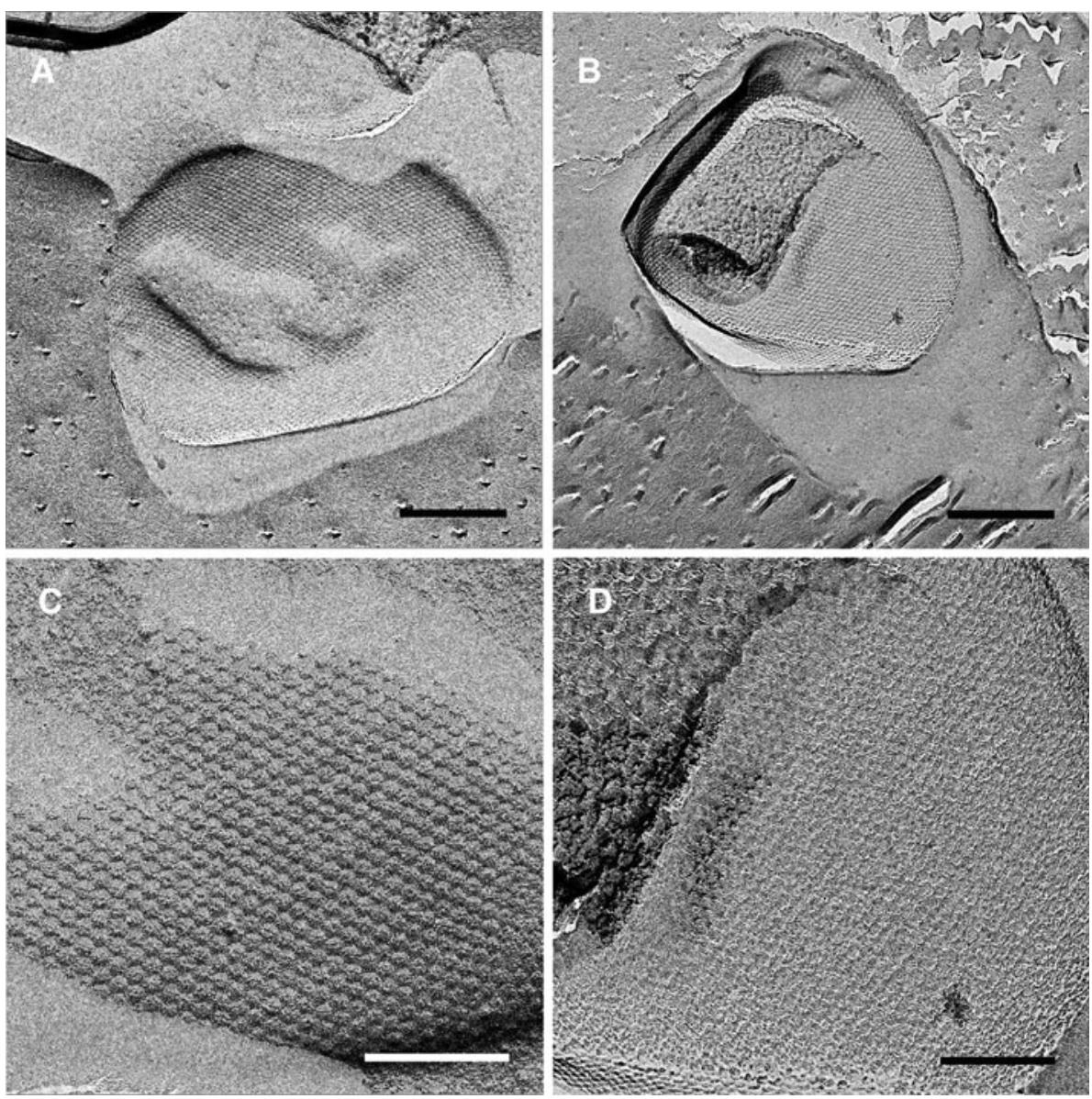

needed for the translocation of sugar binding proteins across the cytoplasmic membrane (Zolghadr et al. 2007). Moreover, in the absence of BasEF, the extracellular binding proteins still bind sugars with high affinity. However, these cells are unable to grow on sugars that for uptake depend on a binding protein that is synthesized with a class III signal peptide, and also sugar transport is impaired. BasE and BasF are homologous to subunits of bacterial type II secretion and type IV pili assembly systems. BasE is a cytosolic ATPase and $\mathrm{BasF}$ is a membrane protein that likely functions to anchor BasE to the membrane. Indeed, BasE and BasF form a complex, but this complex seems labile under the detergent extraction conditions tested. Size exclusion chromatography hints at the presence of a stoichiometric complex rather than a hexameric arrangement of the BasE subunit. Stabilization of the BasEF complex for future structural analysis remains challenging as the complex is readily degraded by endogenous proteases (unpublished results).

An analysis of the ConA purified sugar binding proteins by BN-PAGE and size exclusion chromatography revealed that these proteins are present in a large complex. These complexes could be separated as symmetric peaks on size exclusion chromatography. However, the binding proteins purified from the $\triangle$ basEF strains were present in a similar complex indicating that BasEF is not required for complex formation per se. Possibly, the role of BasEF is to correctly localize the binding protein complexes at the surface layer envelope since some of the binding proteins were found to be associated with the major S-layer protein SlaA. Analysis of the surface envelope by high-pressure freezing and freeze substitution yielded high-resolution images of cytoplasmic membrane, quasi-periplasmic space and S-layer. The TEM images of the S-layer suggested that the Bas system does not just generate a "traditional" filament comparable to flagella or pili, but that it rather is involved in or contributes to shaping the cell envelope. It appears that the deletion of basEF has a disordering effect on the self-assembly of the membrane-integral SlaB (Veith et al. 2009). In this respect, the sugar binding complexes isolated from the cell surface may represent partially dissociated complexes and part of a large native structure in the cell envelope that is a structurally associated with the S-layer. The quasi-periplasmic space of $S$. solfataricus is composed of dense material, most probably glycan chains from lipids, glycosylated proteins and extracellular proteins (see, e.g., the scheme of the S-layer architecture of Halobacterium spp., Kessel et al. 1988), which may be essential for the overall stability of cell morphology and cell wall. Such high density may 
Fig. 8 Electron microscopy of the $\triangle b a s E F$ strain complemented with a plasmid expressing BasEF.

a Transmission electron microscopy of ultra-thin sections of the complemented strain prepared by high-pressure freezing, freeze substitution and embedding in Epon. c Electron micrographs of the freezeetched S-layer of the complemented $\triangle$ basEF strain (b) and (d) represent detailed views of (a) and (c), respectively. Sizes of the bars are in $\mathbf{a}$ and $\mathbf{c} 500 \mathrm{~nm}$ and $\mathbf{b}$ and d $200 \mathrm{~nm}$
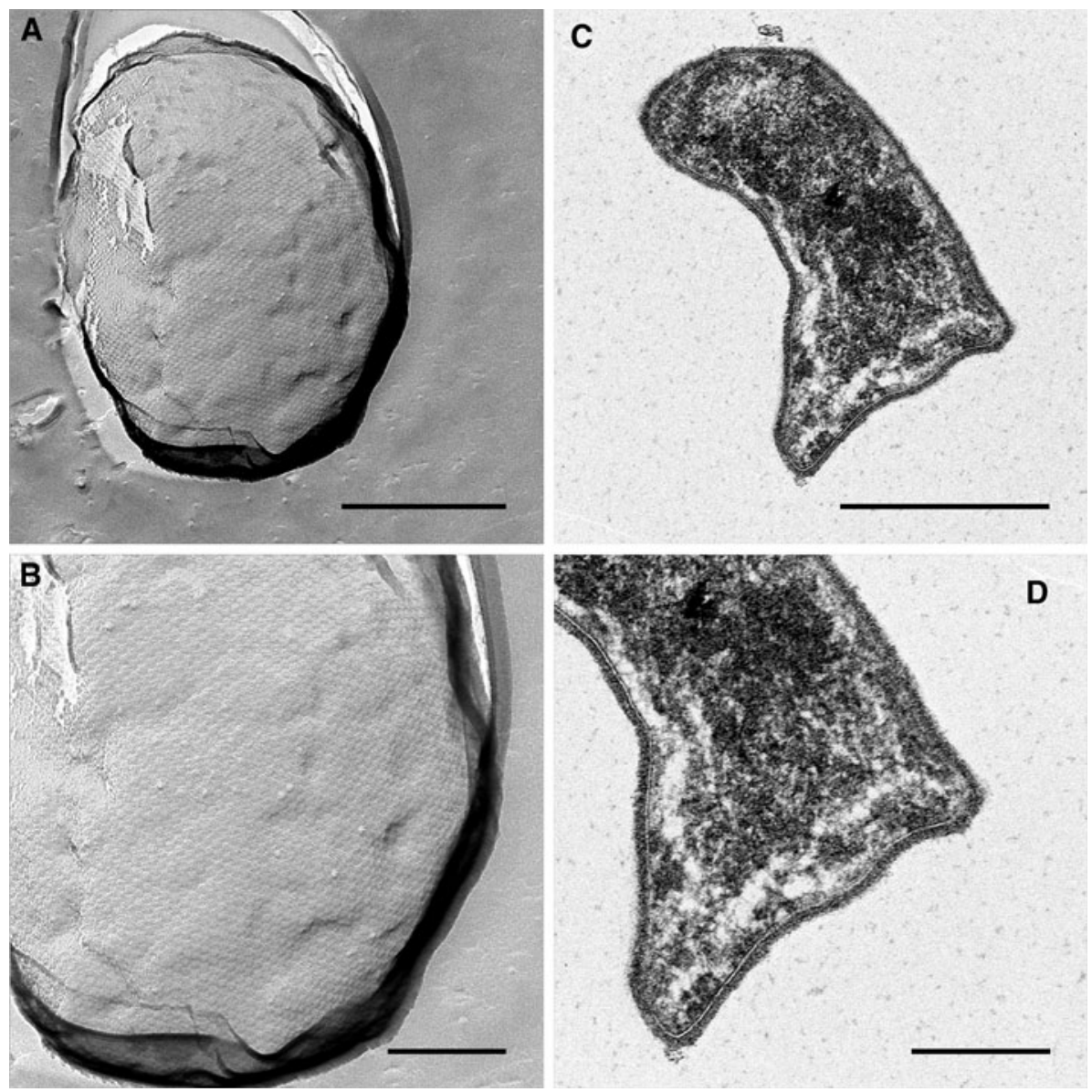

limit the diffusion of extracellular solutes, and assuming that this space amongst others is filled with sugar binding proteins, a specific organization of these binding proteins may facilitate the diffusion of solutes from the outer surface of the S-layer to the cytoplasmic membrane as it is known from Gram-negative bacteria (Brass et al. 1986).

Acknowledgments B.Z. and S.V.A. were supported by a VIDI grant by the Dutch Science Organization (NWO) and intramural funds of the Max Planck Society. The authors wish to acknowledge the technical support by Cordula Neuner. A.K. and R.R. were supported by a grant of the DFG.

Open Access This article is distributed under the terms of the Creative Commons Attribution Noncommercial License which permits any noncommercial use, distribution, and reproduction in any medium, provided the original author(s) and source are credited.

\section{References}

Abendroth J, Murphy P, Sandkvist M, Bagdasarian M, Hol WG (2005) The X-ray structure of the type II secretion system complex formed by the N-terminal domain of EpsE and the cytoplasmic domain of EpsL of Vibrio cholerae. J Mol Biol 348:845-855
Albers SV, Driessen AJM (2002) Signal peptides of secreted proteins of the archaeon Sulfolobus solfataricus: a genomic survey. Arch Microbiol 177:209-216

Albers SV, Driessen AJ (2005) Analysis of ATPases of putative secretion operons in the thermoacidophilic archaeon Sulfolobus solfataricus. Microbiology 151:763-773

Albers SV, Elferink MG, Charlebois RL, Sensen CW, Driessen AJ, Konings WN (1999a) Glucose transport in the extremely thermoacidophilic Sulfolobus solfataricus involves a high-affinity membrane-integrated binding protein. J Bacteriol 181:4285-4291

Albers SV, Konings WN, Driessen AJM (1999b) A unique short signal sequence in membrane-anchored proteins of Archaea. Mol Microbiol 31:1595-1596

Albers SV, Szabo Z, Driessen AJ (2003) Archaeal homolog of bacterial type IV prepilin signal peptidases with broad substrate specificity. J Bacteriol 185:3918-3925

Brass JM, Higgins CF, Foley M, Rugman PA, Birmingham J, Garland $\mathrm{PB}$ (1986) Lateral diffusion of proteins in the periplasm of Escherichia coli. J Bacteriol 165:787-795

Brock TD, Brock KM, Belly RT, Weiss RL (1972) Sulfolobus: a new genus of sulfur-oxidizing bacteria living at low $\mathrm{pH}$ and high temperature. Arch Mikrobiol 84(1):54-68

Craig L, Pique ME, Tainer JA (2004) Type IV pilus structure and bacterial pathogenicity. Nat Rev Microbiol 2:363-378

Elferink MGL, Albers SV, Konings WN, Driessen AJM (2001) Sugar transport in Sulfolobus solfataricus is mediated by two families of binding protein-dependent $\mathrm{ABC}$ transporters. Mol Microbiol 39:1494-1503

Ellen AF, Albers SV, Huibers W, Pitcher A, Hobel CFV, Schwarz H, Folea M, Schouten S, Boekema EJ, Poolman B (2009) Proteomic 
analysis of secreted membrane vesicles of archaeal Sulfolobus species reveals the presence of endosome sorting complex components. Extremophiles 13:67-79

Grogan DW (1989) Phenotypic characterization of the archaebacterial genus Sulfolobus: comparison of five wild-type strains. J Bacteriol 171:6710-6719

Herdendorf TJ, McCaslin DR, Forest KT (2002) Aquifex aeolicus PilT, homologue of a surface motility protein, is a thermostable oligomeric NTPase. J Bacteriol 184:6465

Kessel M, Wildhaber I, Cohen S, Baumeister W (1988) Threedimensional structure of the regular surface glycoprotein layer of Halobacterium volcanii from the Dead Sea. EMBO J 7:15491554

Küper U, Meyer C, Müller V, Rachel R, Huber H (2010) Energized outer membrane and spatial separation of metabolic processes in the hyperthermophilic Archaeon Ignicoccus hospitalis. Proc Natl Acad Sci USA 107(7):3152-3156

Nanavati DM, Thirangoon K, Noll KM (2006) Several archaeal homologs of putative oligopeptide-binding proteins encoded by Thermotoga maritima bind sugars. Appl Environ Microbiol 72:1336-1345

Peabody CR, Chung YJ, Yen MR, Vidal-Ingigliardi D, Pugsley AP, Saier MH Jr (2003) Type II protein secretion and its relationship to bacterial type IV pili and archaeal flagella. Microbiology 149:3051-3072

Planet PJ, Kachlany SC, DeSalle R, Figurski DH (2001) Phylogeny of genes for secretion NTPases: identification of the widespread tadA subfamily and development of a diagnostic key for gene classification. Proc Natl Acad Sci USA 98:2503

Possot O, Pugsley AP (1994) Molecular characterization of pule, a protein required for pullulanase secretion. Mol Microbiol $12: 287-299$
Rachel R, Wyschkony I, Riehl S, Huber H (2002) The ultrastructure of Ignicoccus: evidence for a novel outer membrane and for intracellular vesicle budding in an archaeon. Archaea 1:9-18

Sandkvist M (2001) Biology of type II secretion. Mol Microbiol 40:271-283

Schelert J, Dixit V, Hoang V, Simbahan J, Drozda M, Blum P (2004) Occurrence and characterization of mercury resistance in the hyperthermophilic archaeon Sulfolobus solfataricus by use of gene disruption. J Bacteriol 186(2):427-437

Schoehn G, Quaite-Randall E, Jimenez JL, Joachimiak A, Saibil HR (2000) Three conformations of an archaeal chaperonin, TF55 from Sulfolobus shibatae. J Mol Biol 296:813-819

Sexton JA, Pinkner JS, Roth R, Heuser JE, Hultgren SJ, Vogel JP (2004) The Legionella pneumophila PilT homologue DotB exhibits ATPase activity that is critical for intracellular growth. J Bacteriol 186:1658

Veith A, Klingl A, Zolghadr B, Lauber K, Mentele R, Lottspeich F, Rachel R, Albers SV, Kletzin A (2009) Acidianus, Sulfolobus and Metallosphaera surface layers: structure, composition and gene expression. Mol Microbiol 73:58-72

Yamagata A, Tainer JA (2007) Hexameric structures of the archaeal secretion ATPase GspE and implications for a universal secretion mechanism. EMBO J 26:878-890

Zillig W, Stetter KO, Wunderl S, Schulz W, Priess H, Scholz I (1980) The Sulfolobus-Caldariella Group-taxonomy on the basis of the structure of DNA-dependent RNA-polymerases. Arch Microbiol 125:259-269

Zolghadr B, Weber S, Szabo Z, Driessen AJM, Albers SV (2007) Identification of a system required for the functional surface localization of sugar binding proteins with class III signal peptides in Sulfolobus solfataricus. Mol Microbiol 64:795-806 